\title{
Deep Level Point Imperfections in Semiconductors
}

\author{
Jerzy M. Langer, Warsaw
}

(Institute of Physics, Polish Academy of Sciences)

A standard textbook description of the crystalline solid is a perfect periodic arrangement of atoms. In real life however, all solids are imperfect, and it is the defects that make them useful. It is well known that their presence in insulators at even trace concentrations influences dramatically all their properties - from hardness to conductivity. The latter is just what made semiconductors (bad insulators) the backbone of modern electronics. On the other hand, radiationinduced defects create headaches for nuclear and space engineers.

It is almost impossible even to mention all aspects of defect physics and technology in such a short article, and I shall therefore concentrate on a small, but quite important class of defects in solids: deep level point imperfections in semiconductors. It is one of the branches of semiconductor research which is developing rapidly and is still very far away from saturation.

Defects, perturbing the ideal periodicity of the crystal, must introduce some extra states in the host crystal energy spectrum. Suppose at the beginning that the defect is just a foreign atom (dopant) replacing one of the host atoms and differing from it by a unit valence charge (like arsenic or gallium in silicon). This extra (or deficient) charge is likely to be weakly bound to the impurity atom owing to the host response, and the defect will resemble a hydrogen atom, whose energy levels are scaled down by the host dielectric constant $\varepsilon$, and an effective change $m^{*} / m$ of the electron (or hole) mass. For example, in GaAs considered to be the silicon of the next century - the hydrogen-like donor binding energy $\left\{E_{\mathrm{D}}=13.6\left(m^{*} / m_{\mathrm{e}}\right)^{*} \varepsilon^{-2} \mathrm{eV}\right\}$ is only about $6 \mathrm{meV}$ (a 2000 -fold reduction) and the effective Bohr radius is as large as about $100 \AA$. Such a large spread of the electron wavefunction about the donor makes it fairly insensitive to the chemical nature of the dopant. This is especially evident for the excited p-like states, having a node on the impurity site. Because of that, the Zeeman splitting of these states is often used to obtain a highly accurate determination of the effective mass of the host semiconductor.
When the dielectric screening is not so strong, and especially when the effective mass is not so small, the impurity Bohr radius shrinks and the differences in energy levels introduced by the different isovalent impurities become larger. It may happen, however, that a strong binding impurity (or better to say defect) potential supplemented by the electronphonon interaction will lead to a collapse of the impurity electron wavefunction on to the defect, making it strongly localized with a mean electron radius of the order of the nearest neighbour distance in the host. An almost immediate consequence of this is a sinking of the defect levels (although not always) towards the centre of the semiconductor gap. At this stage a fairly simple hydrogen effectivemass model description tends to fail and a more complex description taking into account not only the detailed defect chemistry, but especially the arrangement of all neighbour atoms around the defect becomes necessary. This becomes a formidable task and is solved satisfactorily in only very rare cases. Unfortunately, most defects fall into this category, and this is why for many years deep level defect research could hardly be considered to be an exact science.

A big change started in the mid-seventies when major progress was made in semiconductor technology, computational techniques for the microscopic theory of point-defects in semiconductors and, especially, in the development of highly complex characterization techniques. They allow us now to trace even single-defect recombination processes as well as single-defect imaging.

Some five years ago, Paul Dean reviewed 1) the basic properties of "deeplevels" in semiconductors. I shall concentrate, therefore on those aspects which have undergone the most rapid development in recent years, pointing out also how "deep" we are and what the problems are that still await a satisfactory explanation.

\section{Signatures of Deep-Level Centres}

What are the properties that characterise most the deep-level defects? Most obvious is the appearance of energy levels whose position and sequence depart strongly from the predictions of a scaled hydrogenic formula. Less obvious, but very typical, is the lifetime shortening of the non-equilibrium minority carriers due to the presence of deeplevel traps. The reason is the exponential increase of recombination rate on the distance of the recombination level from the band edges. Thus even a trace concentration of such traps may fully control the lifetime characteristics of a semiconductor. It was indeed one of the main sources of trouble in the early years of the semiconductor industry. Deeplevel defects may also control the Fermilevel position. This property is currently employed in the growth of semi-insulating III-V compounds like GaAs or InP, used as substrates in semiconductor devices. To obtain the insulation, background doping by shallow impurities must be generally low. For lifetime control, it is not necessary.

Deep-level defect centres may possess several charge states, indicating that many-electron effects contribute to the associated energy spectra and recombination processes. Another characteristic property of these centres is a much larger vibronic coupling than occurs in the delocalized shallow impurity states. The delocalized states couple predominantly to the optical phonons (the so-called Frohlich polar coupling), while those which are localized, predominantly to large k-vector acoustic modes. It is a consequence of the much stronger dependence of the electronphonon coupling parameter on the inverse of the bound carrier radius a for the acoustic phonons $\left(1 / a^{2}\right)$ compared with the optical phonons (1/a). All these phenomena are due to the electron localization at deep-level centres ${ }^{2}$ ).

It was noted some time ago that all defect states are either delocalized or highly localized. The discontinuity in localization results, as shown by Toyozawa and Rashba ${ }^{3}$ ), from the competition between the long-range (Coulombic) and short-range (local defect potential or acoustic phonon coupling) forces. In the defect energy functional, the first leads to an attractive term proportional to $1 / a$, while the second, being proportional to a local charge density, gives the 
attractive term proportional to $1 / a^{3}$. Such a functional produces either delocalized or highly localized states with sometimes an energy barrier between them. This barrier leads to a metastability of one of the two states and has been called by Toyozawa the extrinsic (i.e. defect-induced) self-trapping by analogy to the intrinsic self-trapping associated with polarons or excitons. The occurrence of such metastable states is now a well established characteristic of deep-level defects. Another consequence of the general theory of defects, namely the possibility of binding carriers by the same centre in either highly localized or delocalized effective-mass orbits has been finally proven just recently ${ }^{4}$ ).

Defects strongly coupled to the lattice exhibit many interesting phenomena. Presumably the most unexpected has been the occurrence of the recombination enhanced defect reactions (REDR) $\left.{ }^{2}\right)$. It was found that in conditions far from equilibrium (for example in the presence of a large concentration of non-equilibrium carriers injected into a p-n junction) the activation energy for the defect dissociation or even diffusion becomes smaller and the process can become athermal. This may happen if the recombination process at the defect is non-radiative and the released energy is used to promote the defect reaction. A more likely source of REDR is, however, a lowering of the barrier for dissociation (or other defect reaction) if the defect is in an excited state. Such a situation has been unambiguously proved to occur in a process of defect formation during recombination of excitons in ionic crystals. In semiconductors a direct proof is still lacking.

\section{Many Electron Effects}

Defects can often bind more than just one electron (or hole). A good example is to be found in chalcogens $(0, S$, etc. $)$ in Si. Being substitutional impurities they act as double donors. In principle they should be analogous to $\mathrm{He}$ atoms, but in practice they are not. Another example of a multicharge centre is a vacancy in a semiconductor. Although all these defects are truly localized, the electronelectron interaction is nevertheless much smaller than in the free atoms. This is why the different charge states are separated by only a fraction of an $\mathrm{eV}$. The difference, called $U$, is the sum of a positive electron repulsion term and a negative term due to the electron-phonon coupling (e.g. the Jahn-Teller stabilization energy). If the vibronic component is larger than the electronic, $U$ becomes negative. Then the defect state $D^{\prime}$ is no longer a thermodynamically stable charge state, and all defects must spontaneously disproportionate into an equal number of species of higher and lower oxidation states (i.e. $\mathrm{D}^{i+1}$ and $\left.\mathrm{D}^{i-1}\right)$. Such a phenomenon is well known in the chemistry of metal ions in liquid solutions, and may be the mechanism through which electrons form Cooper pairs in the new high $T_{c}$ superconducting oxides. The first unambiguously identified negative- $U$ centre in a semiconductor was a vacancy in $\mathrm{Si}$, for which the first donor level $(0 /+)$ having one more electron than the second $(+/++)$ lies inverted below it. It should be noted that the negative $U$ behaviour of a silicon vacancy was first predicted in a first principle calculation made by $\mathrm{G}$. Baraff and coworkers from Bell Laboratories and almost immediately confirmed in a tricky experiment made by the group of G. Watkins from the Lehigh University, USA ${ }^{5}$ ).

For most defects in semiconductors, the electronic part of $U$ is rather small (a small fraction of an eV). The only exceptions are transition metal impurities, a group of defects which has attracted a lot of attention recently. Experimentally, they are the centres best identified in semiconductors owing to their unfilled 3d-shell, giving a clear chemical signa-
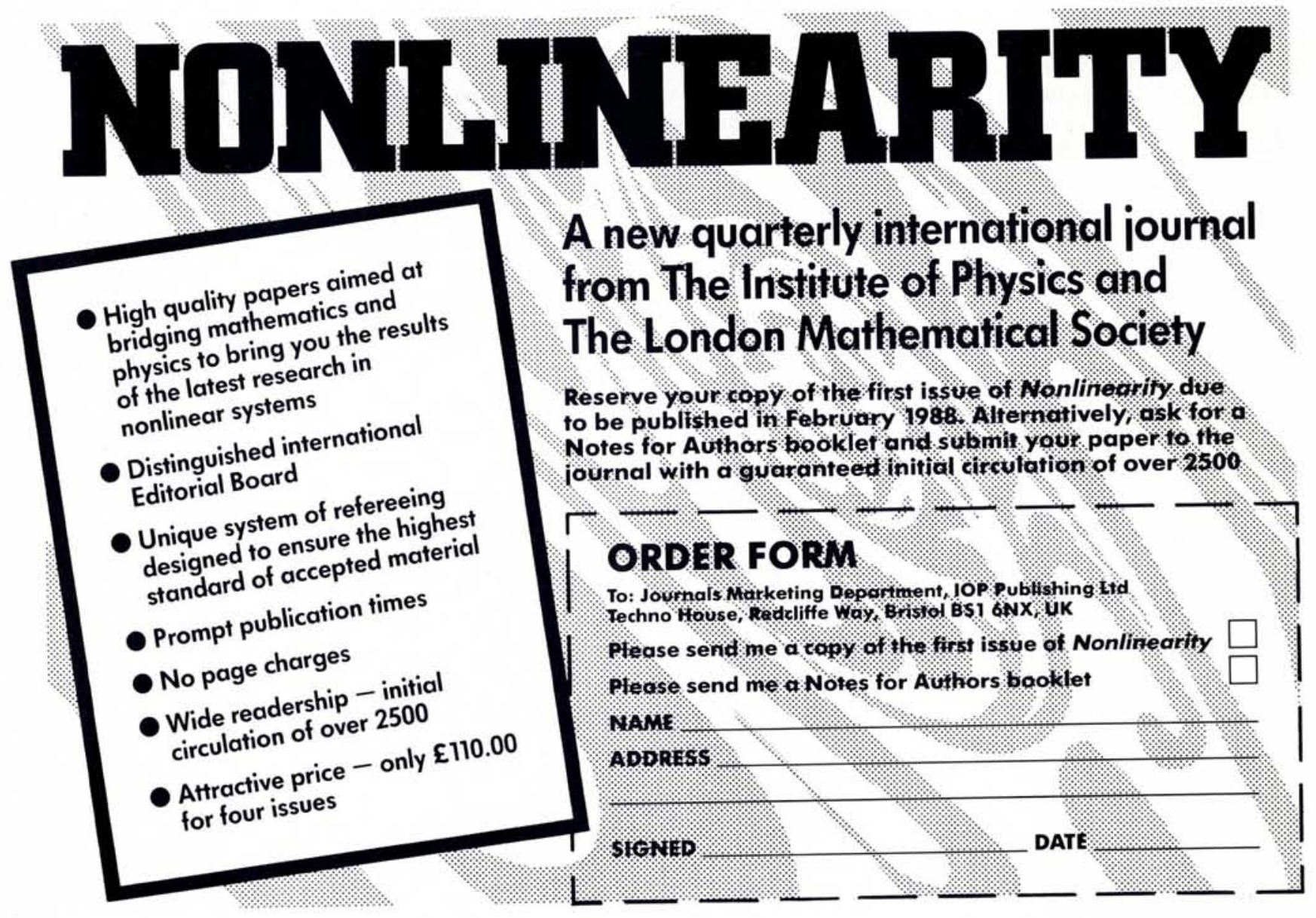


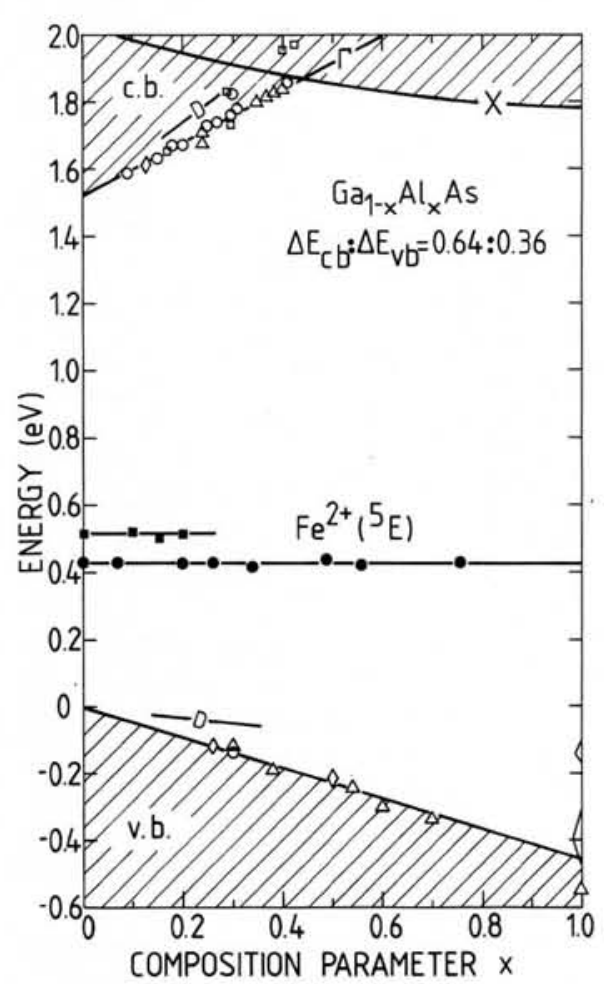

The $\mathrm{Ga}_{1-\chi} A \mathrm{I}_{x}$ As band edges compositional evolution as obtained assuming that the $\mathrm{Fe}^{2+}$ acceptor level may be used as a reference (internal "vacuum level"). The agreement between the predicted slopes and measurements of band edge discontinuities in GaAs/GaAlAs heterostructures (open points) is striking (see ref. 7 for further details).

ture in all spin-sensitive experiments. The theory, although very much progressing in the last few years ${ }^{6}$ ), is still in its evolutionary stage. It must explain a puzzling dichotomy of the properties of this group of centres. Some of them (like a multitude of the charge states in a gap, the relatively large crystal-field splittings or the significant reduction of the spinorbit coupling) indicate strong hybridization of the impurity d states with the host and a reduction of the electronelectron interaction, while others (like the atomic-like multiplet structure of the impurity levels, a strong $d^{5} / d^{4}$ stabilization and relatively small Jahn-Teller effects) may indicate the opposite. The source of this dichotomy, as suggested recently by Zunger ${ }^{6}$ ), is a much weaker screening of the exchange attraction compared with the Coulomb repulsion.

A still relatively large $U$ in this group of impurities explains a bizarre connection discovered recently between the transition-metal energy levels in semiconductors and the energy-band offsets in semiconductor heterojunctions ${ }^{7}$ ). It was found that these levels behave as a reference for the semiconductor bands. The difference in their energy position in the two isovalent semiconductors provides a direct estimate for the valence (or conduction) band offset in a heterojunction made of this pair of semiconductors (Fig. 1).

\section{Research Trends and Perspectives}

One of the strongest driving forces for research in the field is undoubtedly the semiconductor industry. A further miniaturization of solid state devices will exert even stronger pressure, since the role of deep-level defects introduced purposely, or already existing in the material or even introduced during processing, increases dramatically with a reduction in the active volume of a device. The reason is the control they exercise over lifetime in concentrations that can be extremely low. Consequently, the inevitable variations in density that occur, will be reflected in wide variations in the dynamic properties of devices as they become smaller.

A key question addressed to physicists studying the properties of deeplevel centres concerns the microscopic nature of defects influencing properties of the host materials as well as of devices made either on or from them. Identification is therefore the key problem. In spite of a very large effort there are only very few defects, whose chemical identity and dynamic properties have been determined with satisfactory precision. Unfortunately no single tool or analytic prescription on how to achieve this goal exists. Usually many different techniques combined with strong theoretical effort must be used. Bulk measurements like Hall effect have been almost replaced by much more sensitive junction techniques. Also microwave spin resonance techniques like ESR or ENDOR have successfully been combined with optical excitation. These techniques adopted largely from atomic physics have become the main identification tools ${ }^{8}$ ).

Substantial progress in the microscopic theory of defects has made theoretical calculations and modelling a true and indispensable partner in defects research. The best example of this interplay has already been mentioned in the context of a negative- $U$ silicon vacancy. One of the preconditions of a successful attempt at a theory of deep-level centres is the availability of a comprehensive description of the host semiconductor: its band structure and the crystal structure. Such a stage has been achieved just recently owing mainly to progress in computational tools. It is interesting to note, however, that in parallel with weighty computational efforts, much simpler semi-empirical models have been recently developed to track the chemical trends $\left.{ }^{10}\right)$. Their predictive credibility could not however, have been tested, without these much more elaborate and less transparent supercomputer calculations.

\section{REFERENCES}

1. Dean P.J., Europhys. News 14 (1983) 7, 9. 2. Langer J.M., Lecture Notes in Physics 122 (1980) 123; J. Phys. Soc. Japan 49 Suppl A (1980) 207; and Rad. Effects 72 (1983) 55. 3. Toyozawa J., Physica 116 B (1983) 7 and Semicond. and Insul. 5 (1983) 175.

4. Dmochowski J.E., Langer J.M., Kaliński Z. and Jantsch W., Phys. Rev. Lett. 56 (1986) 1735.

5. Watkins G.D., Festkörperprobleme 24 (1984) 163.

6. Vogl P., Festkörperprobleme 25 (1985) 563 and Zunger A., Solid State Phys. 39 (1986) 275.

7. Langer J.M. and Heinrich H., Phys. Rev. Lett. 55 (1985) 1414; Heinrich $\mathrm{H}$. and Langer J.M., Festkörperprobleme 26 (1986) 251; and Tersoff J. and Harrison W., Phys. Rev. Lett. 58 (1987) 2367.

8. Lannoo M. and Bourgoin J., Point Defects in Semiconductors (Springer Verl., Berlin) Vol I 1981 and Vol II 1983.

9. Scheffler M., Festkörperprobleme 22 (1982) 115.

10. Lannoo M., J. Phys. C 17 (1984) 3137 and Acta Phys. Polon. (in print); Vogl P., Adv. Electronics and Electron Phys. 62 (1984) 101.

A most recent review of various aspects of the deep-level research can be found in the book Deep Centers in Semiconductors, ed. S. Pantelides (Gordon and Breach, New York) 1986 as well as in a long review by Blakemore J.S. and Rahimi S. in Semiconductors and Semimetals 20 (1984) 233. Also the Proceedings of the biannual conferences on Defects in Semiconductors (the last published in Mat. Sci. Forum, Vols. 10-12 (1986)) are excellent sources.

\section{AMP BOARD}

Professor J. Los has retired as Secretary of th 2 Atomic and Molecular Physics Division and at the last Board meeting held in Brighton in July he was warmly thanked for his generous help. At the same meeting it was suggested that Professor $\mathrm{H}$. Erhardt of Kaiserslautern University (FRG) be asked to serve as interim Secretary pending the next election of Board members that will take place in 1989. Prof. Erhardt has accepted and agreed to bring his experience and judgment to the services of the Board until 1989.

The next General Conference of our Division, ECAMP III, will be held in Bordeaux, France, in the Spring of 1989 and colleagues from the Soviet Union have agreed to undertake the organisation of ECAMP IV in Riga some time in the late Spring of 1992.

F.A. Gianturco

(Chairman of the AMP Division) 